\title{
Temporal Modeling of Link Characteristic in Mobile Ad hoc Network
}

\author{
Jyoti Prakash Singh ${ }^{1}$ and Paramartha Dutta ${ }^{2}$ \\ ${ }^{1}$ Dept. of Information Technology, National Institute of Technology, Patna, Bihar, India \\ ${ }^{2}$ Dept. of Computer and System Sciences, Visva-Bharati University, West Bengal, India
}

\begin{abstract}
Ad hoc network consists of a set of identical nodes that move freely and independently and communicate among themselves via wireless links. The most interesting feature of this network is that they do not require any existing infrastructure of central administration and hence is very suitable for temporary communication links in an emergency situation. This flexibility, however, is achieved at a price of communication uncertainty induced due to frequent topology changes. In this article, we have tried to identify the system dynamics using the proven concepts of time series modeling. Here, we have analyzed variation of link utilization between any two particular nodes over a fixed area for different mobility patterns under different routing algorithm. We have considered four different mobility models - (i) Gauss-Markov mobility model, (ii) Manhattan Grid Mobility model and (iii) Random Way Point mobility model and (iv) Reference Point Group mobility model. The routing protocols under which, we carried out our experiments are (i) Ad hoc On demand Distance Vector routing (AODV), (ii) Destination Sequenced Distance Vector routing (DSDV) and (iii) Dynamic Source Routing (DSR). The value of link load between two particular nodes behaves as a random variable for any mobility pattern under a routing algorithm. The pattern of link load for every combination of mobility model and for every routing protocol can be well modeled as an autoregressive model of order $p$ i.e. $\operatorname{AR}(p)$. The order of $p$ is estimated and it is found that most of them are of order 1 only.
\end{abstract}

Keywords: ad hoc network, mobility modeling, link load, time series analysis, autoregressive modeling, autocorrelation, white noise

\section{Introduction}

Multi-hop wireless networks [1,2], commonly referred to as ad hoc wireless networks do not require a fixed infrastructure because a mobile node can relay packets to another node without using base stations. The nodes are mobile and changing locations regularly. A node in mobile ad-hoc network (MANET) is moving and its neighborhood varies constantly with time and place. Due to change of this neighborhood the link between two nodes is sometimes available and sometimes not. When the link is up, the number of packets transmitted through that link varies with time. If the link is down, all packets sent through that link are lost. The number of packets transmitted through a link at a particular instance indicates the congestion of that link and, in this article, we term it as link load. The link load is an important index used for congestion control, QoS routing etc. In this article, we have modeled the variation of number of packets transmitted through a particular link between two nodes over time. If we look at the previous link loads of a particular link, we may find that variation of link load shows a specific pattern and this pattern may be modeled in terms of time series. This time series modeling may be used for the purpose of prediction of future link loads. The predicted values of link loads may help us in designing QoS routing algorithms. Let $\mathrm{N}=\left\{\mathrm{N}_{i} \mid 1 \leq i \leq n\right\}$ represents the collection of nodes in the system. A duplex link $\mathrm{L}^{i j}$ is formed between two nodes say $\mathrm{N}_{i}$ and $\mathrm{N}_{j}$ if and only if $\mathrm{N}_{i}$ is neighbor of $\mathrm{N}_{j}$ and $\mathrm{N}_{j}$ is neighbor of $\mathrm{N}_{i}$. The link load $\mathrm{LL}_{t}^{i j}$ of a link between node $\mathrm{N}_{i}$ and $\mathrm{N}_{j}$ is changing constantly with time $t$. At every instant some new nodes are coming into the transmission range of $\mathrm{N}_{i}$ and $\mathrm{N}_{j}$ whereas some old neighbor nodes are leaving the transmission range of $\mathrm{N}_{i}$ and 
$\mathrm{N}_{j}$ thereby leading to change in the load of the link. Say, link $\mathrm{L}^{i j}$ has $\mathrm{LL}_{t}^{i j}$ number of packets at $t$ instance of time. After a small duration of time say $\Delta t$ unit i.e. at time $t+\Delta t$, this number becomes $\mathrm{LL}_{t+\Delta t}^{i j} . \mathrm{LL}_{t+\Delta t}^{i j}$ is a function of the previous link load $\mathrm{LL}_{t}^{i j}, \mathrm{LL}_{t-\Delta t}^{i j}$, and so on down to some early lags. The reason is that some old nodes have left, some new nodes have come into the transmission range with some old nodes still remaining. The link load is highly dependent upon the mobility pattern $[3,4]$ followed by node $\mathrm{N}_{i}, \mathrm{~N}_{j}$ and their surrounding nodes and the routing protocol used. The number of control packets sent due to use of reactive and proactive routing algorithm is different. We define $\mathrm{LL}_{t+\Delta t}^{i j}$ as a function of $\mathrm{LL}_{t}^{i j}, \mathrm{LL}_{t-\Delta t}^{i j}, \mathrm{LL}_{t-2 \Delta t}^{i j}$ and so on. $\mathrm{LL}_{t+\Delta t}^{i j}=\mathrm{f}\left(\mathrm{LL}_{t}^{i j}, \mathrm{LL}_{t-\Delta t}^{i j}, \cdots \mathrm{LL}_{t-p \Delta t}^{i j}\right)$ for some function characteristic $f$ and integer $p$ such that $1 \leq p<\infty$. The autocorrelation properties of LL may also depend on speed and transmission range.

Currently, we have considered four mobility models - (i) Gauss Markov mobility [5], (ii) Manhattan Grid Mobility, (iii) Random Way Point mobility [6, 7] and (iv) Reference Point Group mobility [8] models for our experiment. A survey of the most frequently used mobility models is presented in [3]. The main motivation of the present work is to find a relationship among the link loads values of a particular link LL ${ }^{i j}$ between two nodes $\mathrm{N}_{i}$ and $\mathrm{N}_{j}$ in an ad hoc network. This information can be utilized in other areas such as augmenting the performance of existing routing protocols to include QoS parameters. However, these issues are kept out of the purview of present endeavor in order to avoid shift in the focus of the present article.

The rest of this paper is organized as follows. Section 2 contains a brief survey of the relevant works. Section 3 covers our proposal for using autoregressive model of order $p(A R(p))$ and the relevant discussion. In Section 4 , we provide the simulation results to supplement our proposal of modeling the link load dynamics using $A R(p)$ model. Section 5 describes the techniques to find the order $p$ of $A R(p)$ model. The forecast values to the AR model are also described in that section. In Section 6, we conclude and suggest future directions.

\section{Related Works}

MANETs are expected to be deployed in myriads of scenarios having complex node mobility and connectivity dynamics. For example, in a MANET on a battlefield, the movement of the soldiers will be influenced by the commander. In a city-wide MANET, the node movement is restricted by obstacles or maps. The node mobility characteristics are very application specific. Widely varying mobility characteristics are expected to have a significant impact on the performance of the routing protocols like DSR [9], DSDV[10] and AODV[11]. Bai et al. [12] proposed a framework for analyzing the Impact of Mobility on the Performance Of RouTing protocols in Adhoc NeTworks abbreviated as (IMPORTANT) framework. Through this framework they illustrated the importance of mobility modeling in routing performance and understanding the mechanism of ad hoc routing protocols. They gave a reasonable explanation as to why some routing protocols behave differently under different mobility patterns. To show this difference and supplement their argument, they used a number of useful metrics such as degree of spatial dependence, degree of temporal dependence, connectivity graph, link duration etc. They also give a reasonable explanation as to why mobility effects routing performance. However they failed to give a satisfactory explanation for why routing protocols behave differently even for the same mobility pattern. Helmy et al. $[13,14]$ tried to answer the question "why routing protocols behave differently even for the same mobility pattern"? They tried to answer this question by decomposing a protocol into its protocol mechanistic building blocks. They used reactive protocols like DSR [9] and AODV [11] to explain this. In their work, they decomposed a protocol into its mechanistic building blocks where each mechanistic building block is used to implement a specific function. Then the effect of different mobility patterns on each building block is evaluated. Their approach provides a methodology to justify the contribution of each mechanistic building block on overall protocol performance under various mobility patterns.

Time series $[15,16]$ modeling is drawing a lot of attention in the modeling of internet traffic, wireless sensor and ad hoc network traffic. Basu et al. [17] model the internet traffic using the Autoregressive Moving Average Model of order 
$p$ and $q(A R M A(p, q))$ model. Using this model, they forecast the traffic which was generated by a TCP source using Fiber Distributed Data Interface (FDDI) protocol. Fiber Distributed Data Interface (FDDI) is a set of ANSI and ISO standards for data transmission on fiber optic lines in a local area network that can extend up to a range of $200 \mathrm{~km}$. The FDDI is a token ring based protocol. They also develop a system to generate synthetic traffic which could be useful for simulation studies of internet traffic and in resource management algorithms.

Singh and Dutta $[18,19]$ pointed out the importance of neighbor count of a mobile node and modeled that information using autoregressive model. They showed through extensive simulation that the distribution of neighbor count of a node $N_{i}$ under a threshold value of speed, range and sampling time for different mobility models are well correlated and can be represented by $A R(p)$ model for suitable choice of $p$. However, beyond that threshold value, the autocorrelation of node distribution does not remain significant enough. They also calculated the order of autoregressive model and used that model to predict the neighbor count values.

\section{Proposed Model}

As we pointed out in Section 2, the link count LL ${ }^{i j}$ between node $\mathrm{N}_{j}$ and $\mathrm{N}_{i}$ is changing with time and is a function of the values of link load $L_{L}^{i j}$ at previous instances. Our first concern was to study whether the link load under a specific mobility scenario varies for different routing protocols. If they did so, we tried to find out the amount of variation and correlation for the link load under a specific mobility model using different routing protocol. We have also attempted to answer as to why and how they are varying. We have tried to identify the system dynamics using the proven concepts of time series in this section. A time series $[15,16]$ is a sequence of observations that are arranged according to the time of their outcome. By recording and analyzing the data of a time series, we can gain a better understanding of the data generating mechanism and make a prediction of future values. The main characteristic of a time series is that the data are often governed by a trend and they have periodic components. An important part of the analysis of a time series $[15,16]$ is the selection of a suitable model (or class of models) fitting that data. Naturally, the more appropriate the model selection is, the better is the expected prediction. Lag represents as to how many previous values does the current value of a time series depend on. If the lag value is large, then the current value depends on a large number of previous values. With increase in lag, the number of parameters involved increases too, and as a result the estimation of these parameters becomes time consuming. Nodes change their locations according to underlying mobility models. Based on the physical locations of the nodes, the links are formed. Hence, the mobility models determine how many previous values the current speed and direction should depend on. The link count $\mathrm{LL}^{i j}$ between nodes $\mathrm{N}_{i}$ and $\mathrm{N}_{j}$ is a parameter varying with time that appears to be a suitable candidate for such modeling. The relation of LL with the previous values is also supported with the autocorrelation function or the autocorrelogram of nodes. The experimental data confirms that the autocorrelation is very high at initial lags and is constantly decreasing with higher lags. This implies that the current link count LL can be modeled as a function of values of $L L^{i j}$ on previous instances with a white noise with mean zero and known variance $\sigma^{2}$. This behavior of data can be well represented by an $A R(p)$ model $[15,16]$. The $A R(p)$ model can be defined as

$$
r_{t}=\phi_{0}+\phi_{1} r_{t-1}+\phi_{2} r_{t-2}+\ldots+\phi_{p} r_{t-p}+a_{t}
$$

where $p$ is a non-negative integer and $\phi_{i} \in \operatorname{Re}$ are parameters of the $A R(p) 0 \leq i \leq p$ model. $\mathrm{a}_{t}$ is a white noise sequence with mean zero and variance $\sigma^{2}$ and is independent of $r_{i} \forall i, t-1 \leq$ $i \leq t-p$. This model suggests that the past $p$ values $\mathrm{r}_{t-i}:(i=1, \ldots, p)$ jointly determine the conditional expectation of the past data. The series we used through our experiment is stationary since the following two conditions hold [15]:

1. $\mathrm{E}\left(\mathrm{r}_{t}\right)=\mu$ which is a constant and independent of $t$

2. $\operatorname{Cov}\left(\mathrm{r}_{t}, r_{t-j}\right)=\gamma^{j}$ only depends on $\operatorname{lag} j$, and not on time $t$.

Since the series is stationary, the mean and the variance of this series is governed by the formula

$$
E\left(r_{t}\right)=\frac{\phi_{0}}{1-\phi_{1}-\phi_{2}-\ldots-\phi_{p}}
$$

provided that the denominator is not zero. 
The auto-covariance of the series of equation (1) is given by equation (3) as follows:

$\gamma^{j}= \begin{cases}\phi_{1} \gamma^{j-1}+\phi_{2} \gamma^{j-2}+\ldots+\phi_{p} \gamma^{j-p} & : j=1,2, \ldots p \\ \phi_{1} \gamma^{1}+\phi_{2} \gamma^{2}+\ldots+\phi_{p} \gamma^{p} & : j=0\end{cases}$

The associated polynomial equation of the $A R(p)$ model, called characteristic equation, is given by

$$
x^{p}-\phi_{1} x^{p-1}-\phi_{2} x^{p-2}-\ldots-\phi_{p}=0
$$

Another condition of stationarity is that if all the characteristic roots of equation (4) are less than unity in modulus, then the series $r_{t}$ is stationary [15]. The characteristic equation indicates that the plot of the autocorrelation, known as autocorrelation function, (ACF) of $A R(p)$ model shows a mixture of damping sine and cosine patterns and exponential decays depending on the nature of its characteristic roots. This is in strong conformity with our experimental data. One major hurdle in representing data using $A R(p)$ model is finding the appropriate value of $p$ and solving the AR coefficients. We have used the Yule-Walker equations to determine the AR coefficient for the arbitrary $p$. The Yule-Walker equation in matrix form is given as

$$
\left[\begin{array}{c}
r_{1} \\
r_{2} \\
r_{3} \\
\vdots \\
r_{N}
\end{array}\right]=\left[\begin{array}{cccc}
1 & x_{1} & \cdots & x_{N-1} \\
x_{1} & x_{2} & \cdots & x_{N-2} \\
x_{2} & x_{3} & \cdots & x_{N-3} \\
\vdots & \vdots & & \vdots \\
x_{N-1} & x_{N-2} & \cdots & 1
\end{array}\right] \times\left[\begin{array}{c}
a_{1} \\
a_{2} \\
a_{3} \\
\vdots \\
a_{N}
\end{array}\right]
$$

where $x_{d}$ represents the autocorrelation coefficient at lag d. We can find the $x_{d}$ values by the following method. Let us consider the general $A R(p)$ model once again, as given in equation 1.

$$
r_{t}=\phi_{0}+\phi_{1} r_{t-1}+\phi_{2} r_{t-2}+\ldots+\phi_{p} r_{t-p}+a_{t}
$$

Multiplying both sides of the equation by $r_{t-1}$ gives

$$
r_{t} r_{t-1}=\sum_{t=1}^{p}\left(\phi_{j} r_{t} r_{t-j+1}\right)+r_{t-1} a_{t}+r_{t-1} \phi_{0}
$$

where $j$ and $t$ are term and time indices respectively. Considering expectations on both sides

$$
\begin{aligned}
E\left[r_{t} r_{t-1}\right]= & \sum_{t=1}^{p}\left(\phi_{j} E\left[r_{t} r_{t-j+1}\right]\right) \\
& +E\left[r_{t-1} a_{t}\right]+E\left[r_{t-1}\right] \phi_{0}
\end{aligned}
$$

where the $\phi_{j}$ values are kept outside the purview of the expectation because they are parameters rather than random variables. It is to be noted that $E\left[r_{t-1} a_{t}\right]=0$ and $E\left[r_{t-1}\right] \phi_{0}=0$ because the random perturbation a of the current time is statistically uncorrelated with the previous values of the process. Therefore, we get

$$
E\left[r_{t} r_{t-1}\right]=\sum_{t=1}^{p}\left(\phi_{j} E\left[r_{t} r_{t-j+1}\right]\right)
$$

Next, dividing by $(N-1)$ throughout and using the evenness of the auto-covariance i.e. $c_{1}=c_{-1}$, we get

$$
c_{1}=\sum_{t=1}^{p} \phi_{j} c_{j-1}
$$

Finally, dividing throughout by $c_{0}$, we get

$$
x_{1}=\sum_{t=1}^{p} \phi_{j} r_{j-1}
$$

which gives $x_{1}$ in terms of $1, x_{1}, \cdots, x_{N-1}$. Repeating the same process, we get $x_{2}$ in terms of $x_{1}, x_{2}, x_{3} \cdots, x_{N-2}$ and so on. Finally writing all this together gives the Yule-Walker equation given in equation 5 which can be solved to get the coefficients of $A R(p)$ model. We can also get partial autocorrelation function using YuleWalker equation. To find the order $p$ of the $A R(p)$ model, we have used the partial autocorrelation function (PACF). Finally Akaike Information Criterion (AIC) [16] is used to confirm the choice of $p$ suggested by partial autocorrelation function. The link interference is the total useless signals transmitted by the nodes within their interference ranges. Interference leads to collisions and packet losses, resulting in retransmissions and more energy consumption. It becomes one of the most important factors affecting the performance of networks. As pointed by $\mathrm{Xu}$ et al. [20], the interference range of a mobile node is 1.78 times the transmission range. The nature of interference surely depends on the mobility model under consideration. However, in respect of the present scope of investigation, our suggested model does not incorporate the issue of interference within its purview. 


\section{Simulation Results}

Our first concern was to study whether the link load under a specific mobility scenario varies for different routing protocol. If they do so, we tried to find out the amount of variation and correlation for the link load under a specific mobility model using a different routing protocol. We also attempted to determine why and how they are varying.

We have used Bonn-Motion [21] for generating mobility scenarios. We have generated four mobility patterns with 50 nodes moving in an area of $1000 \mathrm{~m} \times 800 \mathrm{~m}$ for a period of 1000 s. While generating the movement patterns, we ignore the first 3600 seconds of movement and then store the next 1000 seconds of mobility pattern. It has been observed that with the Random Way Point mobility model, nodes have a higher probability of being near the center of the simulation area, while they are uniformly distributed over the simulation area initially. Similarly, in Manhattan Grid Mobility model, all nodes start from $(0,0)$ and then they get distributed over the simulation area. So, we skip 3600 seconds at the beginning to mitigate the boundary effects of node movement simulation. The maximum speed $\mathrm{V}_{\text {max }}$ of a node is set to $5 \mathrm{~m} / \mathrm{s}, 10 \mathrm{~m} / \mathrm{s}$, $20 \mathrm{~m} / \mathrm{s} 30 \mathrm{~m} / \mathrm{s}, 40 \mathrm{~m} / \mathrm{s} 50 \mathrm{~m} / \mathrm{s}$, and $60 \mathrm{~m} / \mathrm{s}$ respectively. The minimum speed $\mathrm{v}_{\min }$ of a node is always set to $0.5 \mathrm{~m} / \mathrm{s}$. The $\mathrm{v}_{\min }$ was set to a positive value because Yoon and Liu [22] proved mathematically that the average speed of the nodes using Random way point mobility model decrease constantly and would eventually reach zero. One of their suggestions for getting rid of this problem was to use non zero minimum speed. This is what we followed here. Gauss Markov mobility model is characterized by the twin parameters viz. speed $s$ and direction $d$. The value of speed and direction at $n^{\text {th }}$ instance is calculated based upon the value of speed and direction at the $(n-1)^{s t}$ instance using equations 12 and 13.

$$
\begin{aligned}
& s_{n}=\alpha_{s_{n-1}}+(1-\alpha) s+q\left(1-\alpha^{2}\right) s_{x_{n-1}} \\
& d_{n}=\alpha_{d_{n-1}}+(1-\alpha) d+q\left(1-\alpha^{2}\right) d_{x_{n-1}}
\end{aligned}
$$

where $s_{n}$ and $d_{n}$ are the new speed and direction at time interval $n$ and $\alpha: 0 \leq \alpha \leq 1$ is the tuning parameter used to vary the randomness. $s$ and $d$ are constants representing the mean value of speed and direction as $n \rightarrow \infty$. $s_{x_{n-1}}$ and $d_{x_{n-1}}$ are random variables from Gaussian distribution. For our experiment, we have taken $\alpha=0.75$ whereas $s_{x_{n-1}}$ and $d_{x_{n-1}}$ are chosen from $\mathrm{N}(0,1)$ distribution. The cbrgen tool which is a part of ns-2 [23] distribution is used to generate Constant Bit Rate traffic for 1000s with 1 packet/sec per source. The number of sources and destinations were chosen randomly by cbrgen tool. We have used ns-2 [23] for network simulation and traces are generated in new trace format. The link load values for each time period are calculated from those trace files using some AWK scripts. The computed values of link load are then taken to Minitab for further analysis. We tested the pattern of link loads for stationarity and then determined the autocorrelation function (ACF) and partial autocorrelation function (PACF).

We have analyzed the link load for four mobility models (i) Gauss Markov, (ii) Manhattan Grid, (iii) Random Way Point [6] and (iv) Reference Point Group Mobility models. We studied the link loads under three different routing protocols (i) AODV, (ii) DSDV and (iii) DSR.

\subsection{Analysis under Gauss Markov Mobility Model}

Our first experiment deals with finding the relationship between the autocorrelation of $L^{i j}$ for Gauss Markov Mobility model for different routing algorithms. As can be seen from Figure 1, the average link load is higher for AODV compared to DSR and DSDV. The operation of both AODV and DSR has two phases: (i) Route setup phase and (ii) Route Maintenance phase. Global flooding is the basic mechanism used to propagate the route request messages for finding a route to destination. DSR uses a non-propagating direct neighborhood inquiry whereas AODV uses expanding ring search before applying global flooding. Both protocols also use caching to reply the route request based on the cached routing information. DSR monitors the link status at the MAC layer whereas AODV uses Hello messages to monitor the link status. The different ways of handling the link status account for the change of different link loads in those protocols. In route maintenance phase, the unstable links are repaired. DSDV is a table driven protocol and hence uses periodic updates to maintain routes. At low speed, 


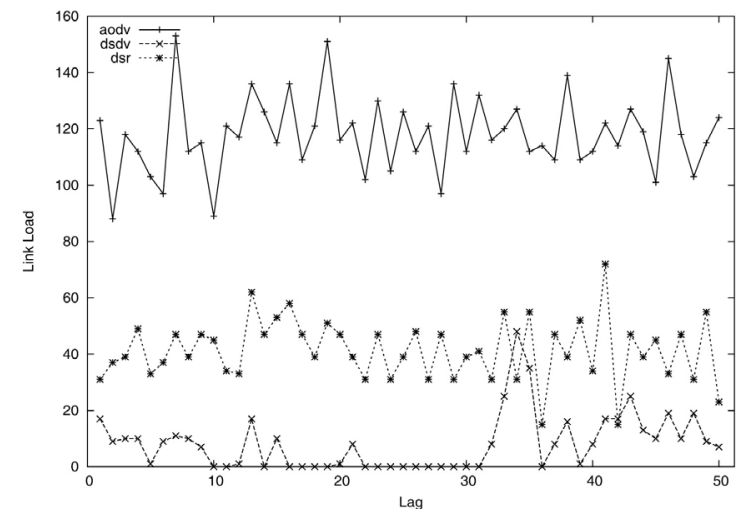

Figure 1. Time series plot of link load for a different routing protocol using Markov Mobility pattern.

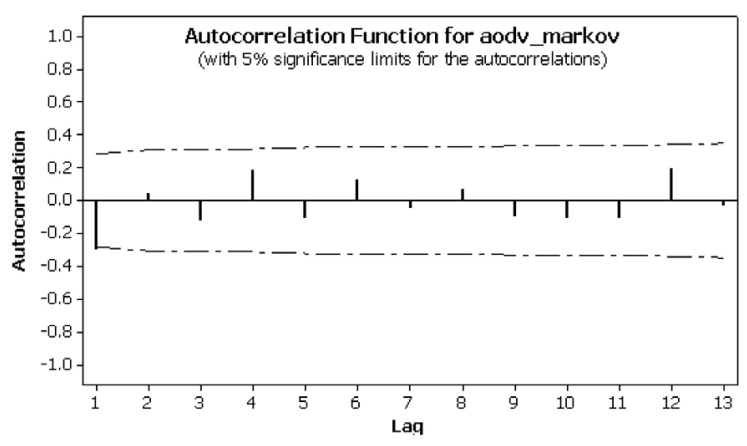

Figure 2. Autocorrelations of link load values for AODV routing protocol using Markov Mobility pattern.

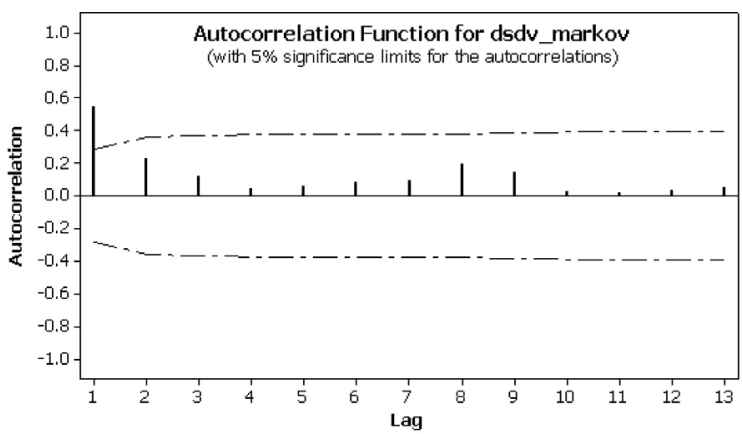

Figure 3. Autocorrelations of link load values for DSDV routing protocol using Markov Mobility pattern.

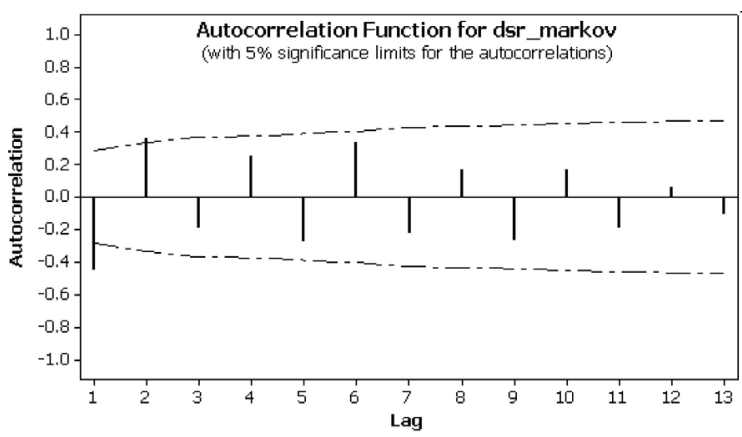

Figure 4. Autocorrelations of link load values for DSR routing protocol using Markov Mobility pattern. the route changes are low and hence DSDV performs better than AODV and DSR. The autocorrelation function of link load values for nodes using AODV [11] routing protocol and moving with Gauss Markov Mobility pattern is shown in Figure 2. Figure 3 illustrates the autocorrelation function of link load values for nodes using DSDV [10] routing protocol and moving with Gauss Markov Mobility pattern. The autocorrelation function of link loads values for nodes using DSR [9] routing protocol and moving with Gauss Markov Mobility pattern is shown in Figure 4. It is evident from Figures 2, 3 and 4 that the Link Load values LL $L^{i j}$ between node $i$ and $j$ show a strong autocorrelation with high peaks at initial lags, but decrease at higher lags. The characteristics of the autocorrelation functions shown in Figures 2, 3, and 4 confirm that the $\mathrm{LL}^{i j}$ between node $i$ and $j$ values for nodes moving under Gauss Markov Mobility model can be fitted to $A R(p)$ model with suitable value of $p$.

\subsection{Analysis under Manhattan Grid Mobility Model}

The second experiment deals with finding the relationship between the autocorrelation of $L^{i j}$ for Manhattan Grid Mobility model for different routing algorithms. As can be seen from Figure 5, the average link load is higher for AODV compared to DSR and DSDV, as was the case with Gauss Markov Mobility model. But this time the differences in the link load values across routing protocols are not so prominent. This is because, in Manhattan Grid Mobility model, the nodes move in a restrictive lane and hence the overhead of link break is comparatively low. The autocorrelation function of link load values for nodes using AODV [11] routing protocol and moving with Manhattan Grid Mobility pattern is shown in Figure 6. Figure 7 illustrates the autocorrelation function of link loads values for nodes using DSDV [10] routing protocol and moving with Manhattan Grid Mobility pattern. The autocorrelation function of link loads values for nodes using DSR [9] routing protocol and moving with Manhattan Grid Mobility pattern is shown in Figure 8. In case of Manhattan Grid Mobility model, the link load values $L^{i j}$ between nodes $i$ and $j$ show a strong autocorrelation with peaks at initial lag, 


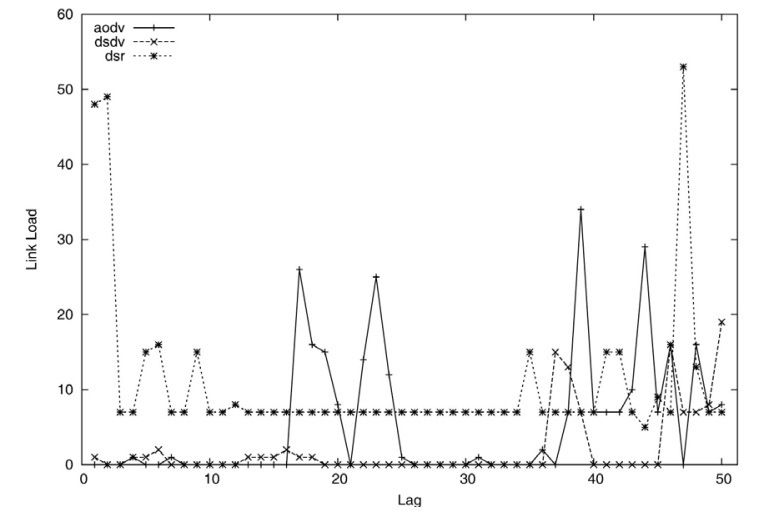

Figure 5. Time series plot of link load for a different routing protocol using Manhattan Mobility pattern.

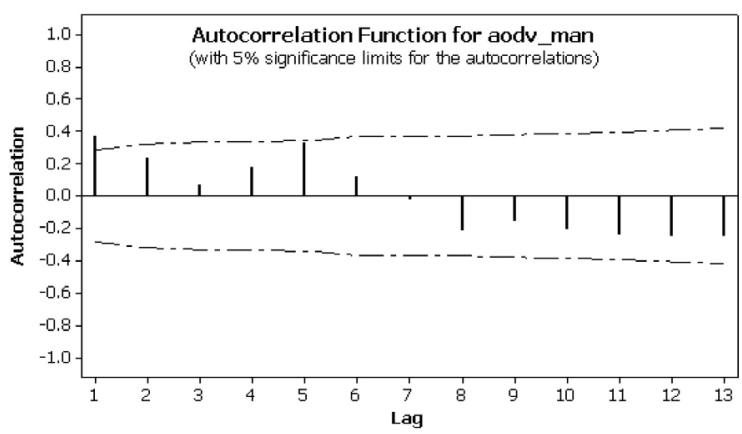

Figure 6. Autocorrelations of link load values for AODV routing protocol using Manhattan Grid Mobility pattern.

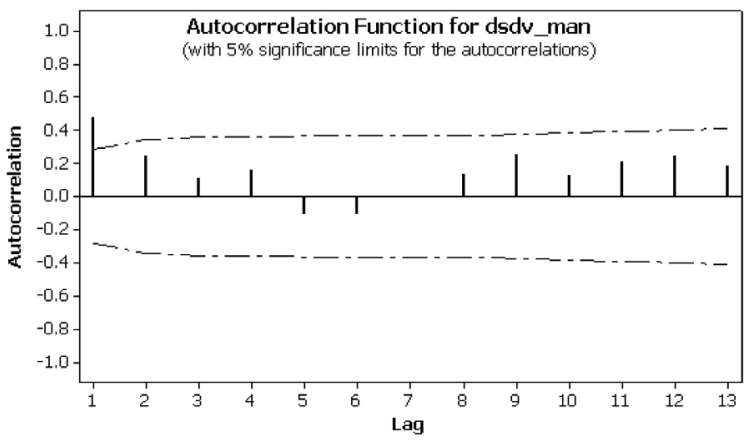

Figure 7. Autocorrelations of link load values for DSDV routing protocol using Manhattan Grid Mobility pattern.

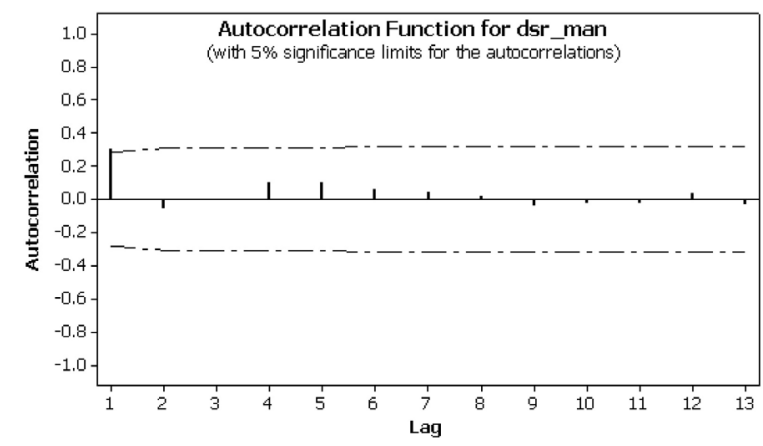

Figure 8. Autocorrelations of link load values for DSR routing protocol using Manhattan Grid Mobility pattern. as shown in Figure 8. Hence, the link load values $\mathrm{LL}^{i j}$ between nodes $i$ and $j$ moving under these mobility models can also be modeled using $A R(p)$ model with suitable $p$.

\subsection{Analysis under Reference Point Group mobility Model}

The third experiment deals with finding the relationship between the autocorrelation of $\mathrm{LL}^{i j}$ for Reference Point Group mobility model for different routing algorithms. As can be seen from Figure 9, the average link load is higher for AODV compared to DSR and DSDV. The autocorrelation function of link loads values for nodes using AODV [11] routing protocol and moving with Reference Point Group Mobility pattern is shown in Figure 10. Figure 11 illustrates the autocorrelation function of link loads values for nodes using DSDV [10] routing protocol and moving with Reference Point Group Mobility pattern. The autocorrelation function of link loads values for nodes using DSR [9] routing protocol and moving with Reference

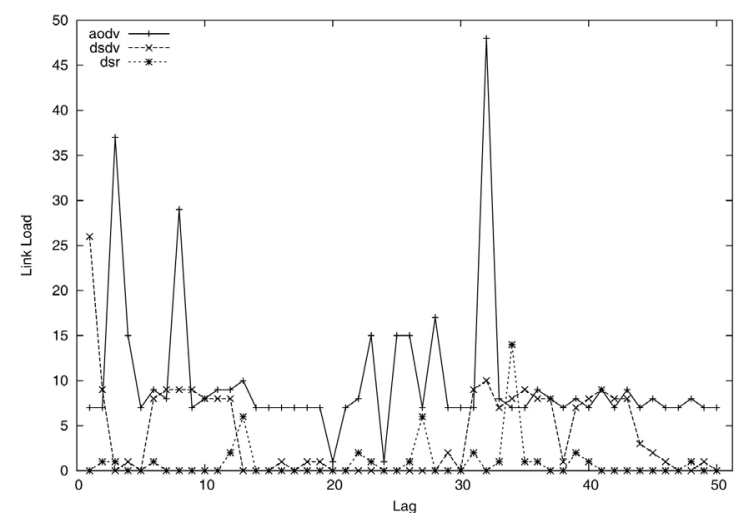

Figure 9. Time series plot of link load for a different routing protocol using RPGM Mobility pattern.

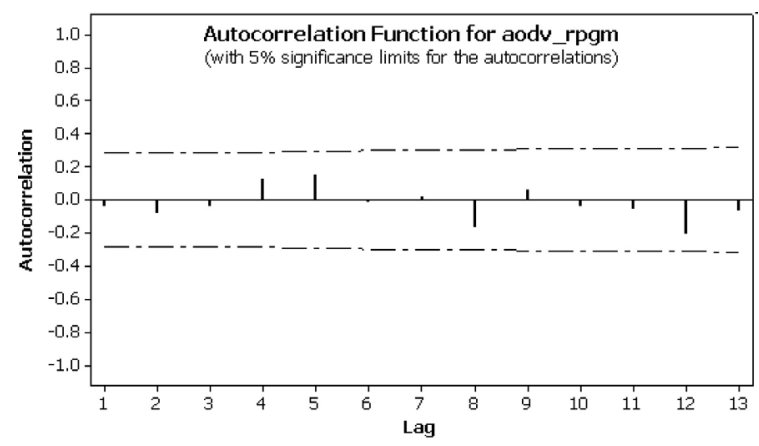

Figure 10. Autocorrelations of link load values for AODV routing protocol using RPGM Mobility pattern. 


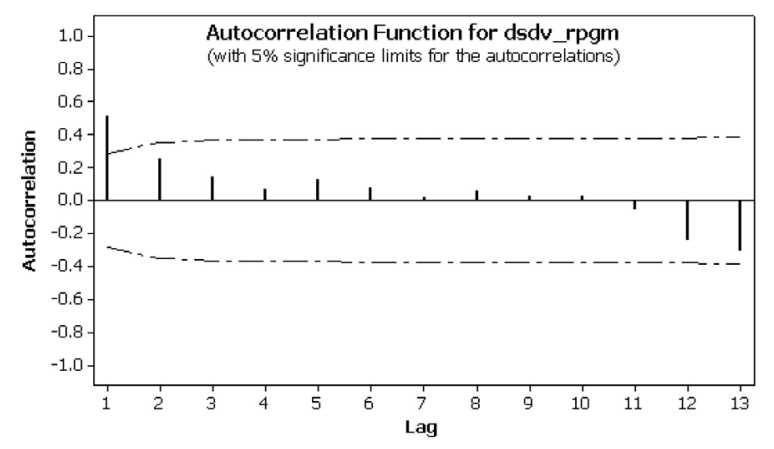

Figure 11. Autocorrelations of link load values for DSDV routing protocol using RPGM Mobility pattern.

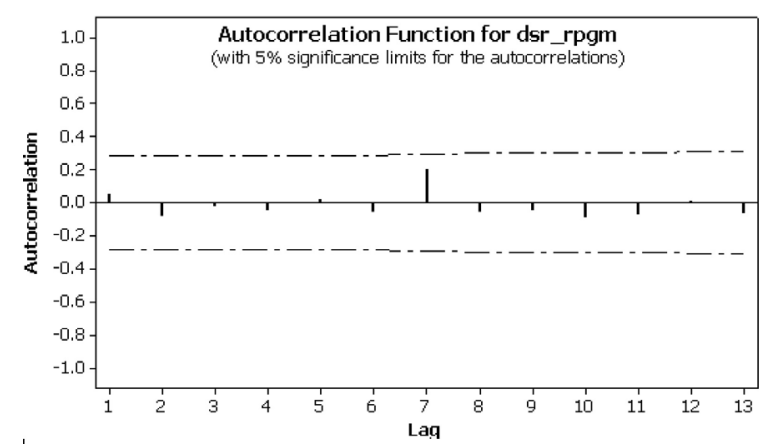

Figure 12. Autocorrelations of link load values for DSR routing protocol using RPGM Mobility pattern.

Point Group Mobility pattern is shown in Figure 12.

\subsection{Analysis under Random Way Point Mobility Model}

The fourth experiment deals with finding the relationship between the autocorrelation of $\mathrm{LL}^{i j}$ for Random Way Point Mobility model for different routing algorithms. As can be seen from Figure 13, the average link load is higher for

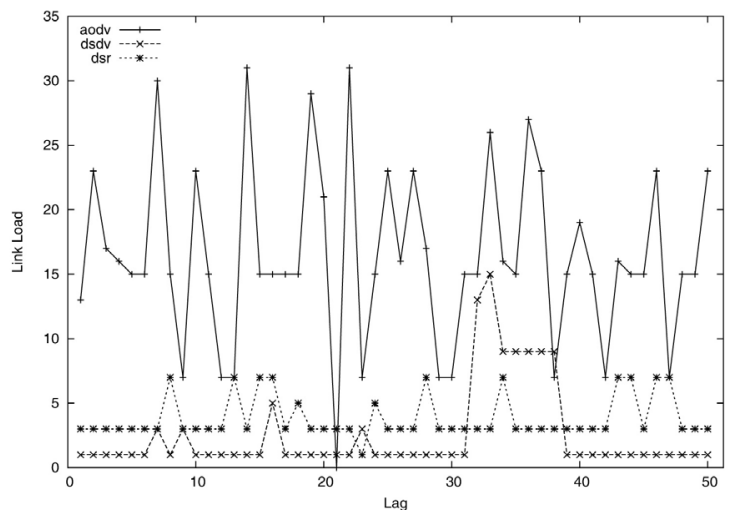

Figure 13. Time series plot of link load for a different routing protocol using Random Way Point Mobility pattern.
AODV compared to DSR and DSDV. The autocorrelation function of link loads values for nodes using AODV [11] routing protocol and moving with Random Way Point Mobility pattern is shown in Figure 14. Figure 15 illustrates the autocorrelation function of link loads values for nodes using DSDV [10] routing protocol and moving with Random Way Point Mobility pattern. The autocorrelation function of link loads values for nodes using DSR [9] routing protocol and moving with Random Way Point Mobility pattern is shown in Figure 16.

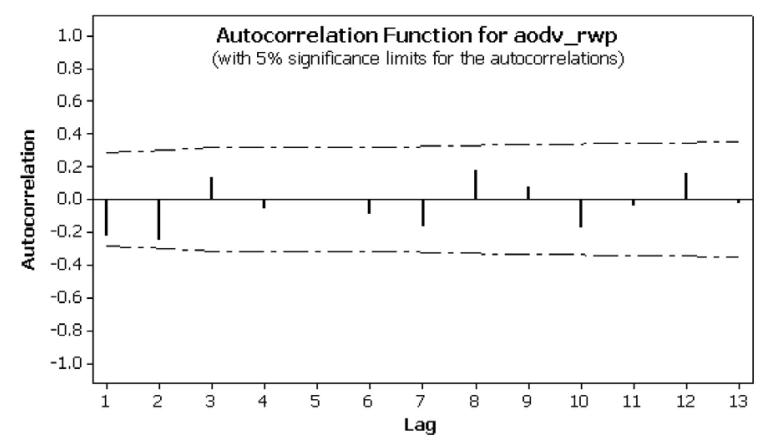

Figure 14. Autocorrelations of link load values for AODV routing protocol using Random Way Point Mobility pattern.

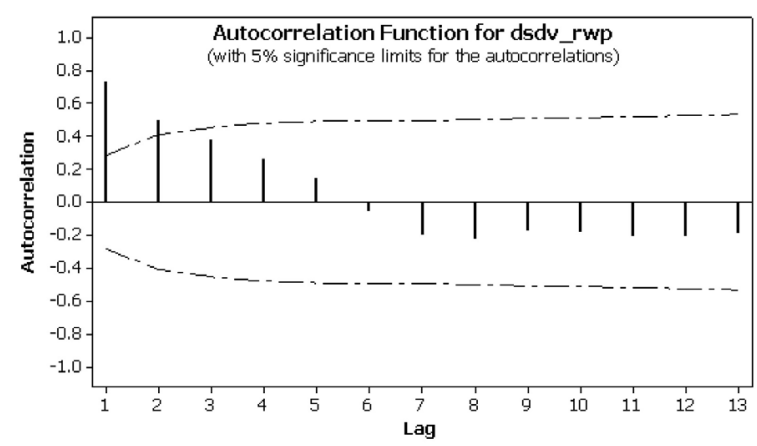

Figure 15. Autocorrelations of link load values for DSDV routing protocol using Random Way Point Mobility pattern.

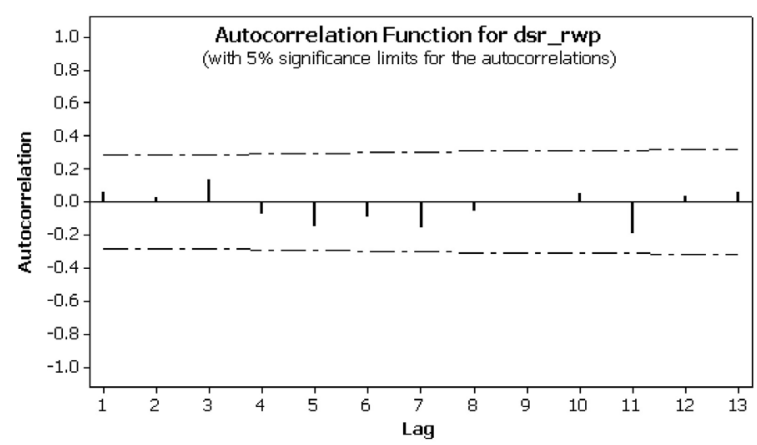

Figure 16. Autocorrelations of link load values for DSR routing protocol using Random Way Point Mobility pattern. 


\section{Discussion}

As evident from the previous simulation results, for a specific transmission range the link load LL data shows strong autocorrelation with high peaks at initial lags which decreases at higher lags. This property of data confirms that it is justified to model the link load LL data with autoregressive model. Autoregressive modeling requires the order $p$ of the model to be determined. In the next section, we outline the process for evaluating the order $p$ of $A R(p)$ model.

\begin{tabular}{|c|c|}
\hline AIC value & Order of AR model \\
\hline \hline 374.794 & 1 \\
\hline 374.627 & 2 \\
\hline 376.592 & 3 \\
\hline 377.215 & 4 \\
\hline
\end{tabular}

Table 1. Finding the order of Autoregressive Model using AIC for link load using DSR routing under Gauss Markov Mobility pattern.

The chosen $A R(p)$ model is then used to predict the next value of the link load.

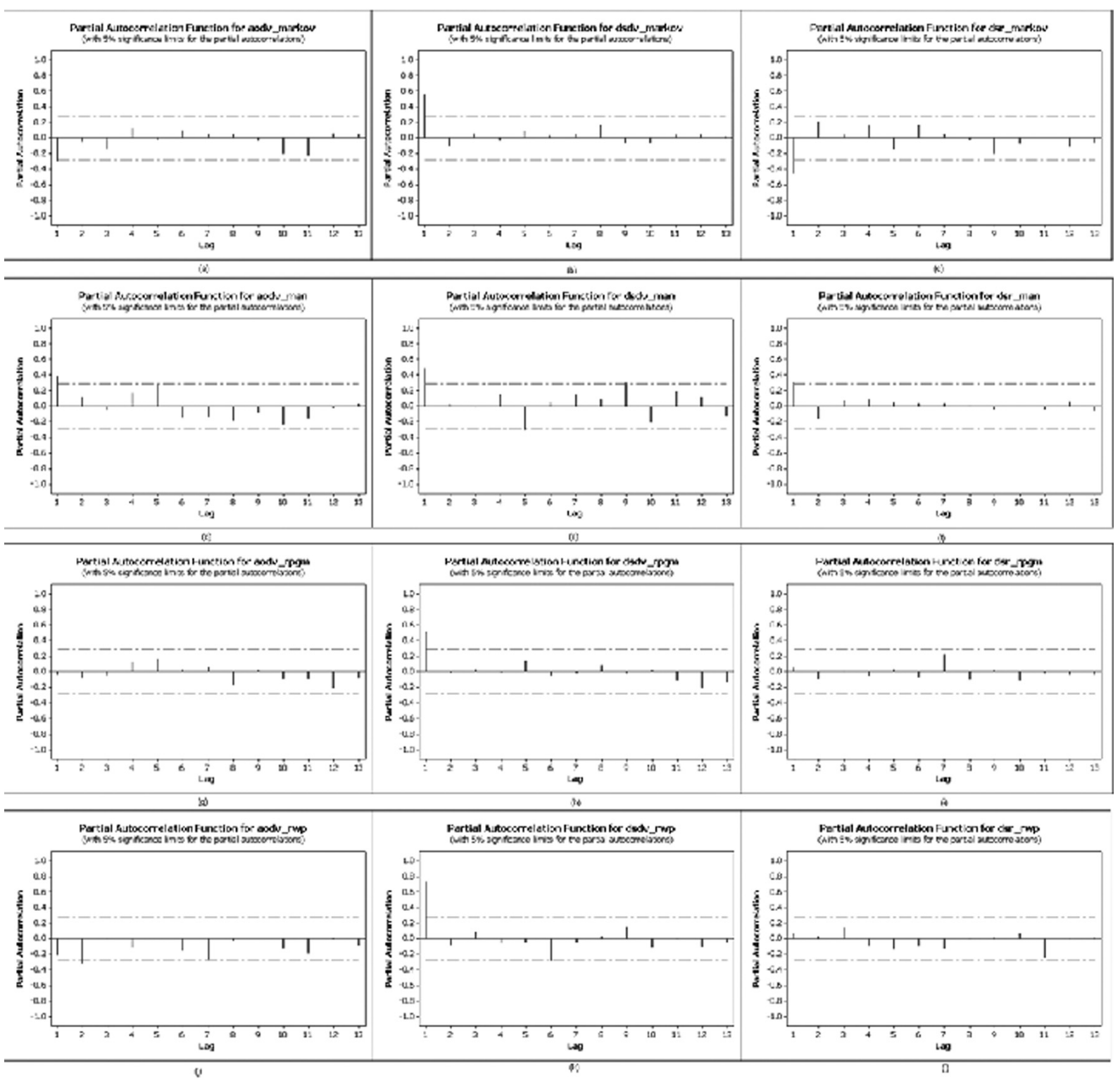

Figure 17. Partial correlation function of link loads using (a) AODV protocol, (b)DSDV protocol, (c)DSR protocol moving under Gauss Markov Mobility pattern, (d)using AODV protocol, (e) DSDV protocol, (f) DSR protocol moving under Manhattan Grid Mobility pattern, (g)using AODV protocol, (h) DSDV protocol, (i) DSR protocol moving under Reference Point Group Mobility pattern, $(\mathrm{j})$ using AODV protocol, (k) DSDV protocol and (1) DSR protocol moving under Random Way Point Mobility pattern. 


\subsection{The Order of AR}

To find out the order $p$ of AR process, we have to determine the PACF, which gives an indication of the probable values of $p$. Then we use AIC to confirm that. The Akaike Information Criterion (AIC) is a way of selecting a model from among a set of models. The chosen model is one that minimize the Kullback-Libler distance metric between the models and the truth. AIC not only rewards goodness of fit, but also imposes a penalty that is an increasing function in respect of the number of estimatable parameters. This penalty discourages over fitting. To apply AIC in practice, we start with a set of candidate models, and then find the models corresponding to AIC values. We identify the model with the minimum AIC value. Models having their AIC values in the range of 1 and 2 of the minimum have substantial support and should be considered in making inferences. This is how we fix order $p$ of our model. The least square method finds the best estimate of unknown parameters, given a specific model. Here, the question is different. Here our problem is of fixing the most suitable model based on available data. Least square regression does not serve the purpose as that offered by AIC. The PACF of the LL values for nodes moving with speed $1.5 \mathrm{~m} / \mathrm{sec}$ is depicted in Figure 17. The AIC values of data set representing link loads LL ${ }^{i j}$ of link between nodes $i$ and $j$ moving under Gauss Markov Mobility model and using DSR as routing protocol are tabulated in Table 1. The PACF of the data set representing link loads $L^{i j}$ of link between nodes $i$ and $j$ moving under Gauss Markov Mobility model and using DSR as routing protocol shown in Figure 17 (c) tells us that this data can be modeled using an $A R(1)$ process. When we find the AIC values, the value is smaller for 2 , but the difference is very low. Thus, it is justified to take the value of $p$ to be 1 . We have used our model to forecast the next LL value. We have selected the link load data set moving under DSR routing protocol and following Gauss Markov Mobility model for forecasting. We have modeled the said data set using autoregressive model of order 1, as discussed in Section 5.1. We used the said model to forecast the next 10 values of the data set. The comparison of forecast and original data obtained is shown in Figure 18. The actual data and the forecast data values are very

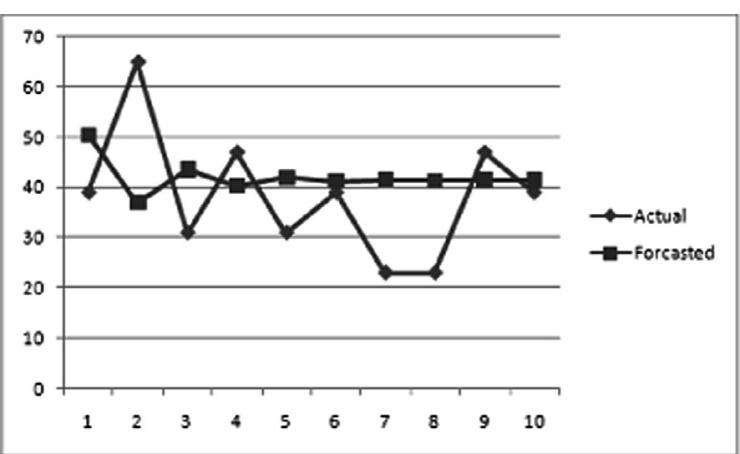

Figure 18. Forecast value of LL made by AR(1) model for DSR routing with Gauss Markov Mobility pattern.

close to each other, as evident from Figure 18. We have formulated the following two hypotheses based on the observed and expected data to validate our point statistically that the forecast values are indeed close to actual value.

$\mathrm{H}_{0}$ : The expected values and predicted values match.

$\mathrm{H}_{1}$ : The expected values and predicted values do not match.

We have used $\chi^{2}$ test to test the hypothesis. The test criterion for $\chi^{2}$ test is $\chi_{0}^{2}=\sum_{i=1}^{n} \frac{\left(O_{i}-E_{i}\right)^{2}}{E_{i}}$. Here $\mathrm{O}_{i}$ refers to the observed values and $\mathrm{E}_{i}$ refers to the expected values. For the data shown in Figure 18, the computed $\chi_{0}^{2}$ value is 6.14 which is much smaller than the critical value of $\chi_{0.05,9}^{2}$ with 9 degrees of freedom at $5 \%$ significance level. Thus the hypothesis that the forecast value is similar to actual value may be accepted with $95 \%$ confidence.

\section{Conclusion}

In this article, we have modeled the link load distribution of a link between two nodes using an Auto-regressive $A R(p)$ model used in stationary time series analysis. We found through our experiments that link load distribution between two nodes for all four mobility models considered in this article are well correlated and can be represented by $A R(p)$ model for suitable value of $p$. We have also predicated the link loads between two nodes in future time frames and found that the prediction is close enough to the real values. These predicated values of link loads may be used for routing with quality of service parameters. It is doubtless that 
transmission delay is caused by the presence of interference. The nature of interference depends on the mobility model under consideration. However, in respect of the present scope of investigation, our suggested model does not incorporate the issue of interference within its purview. Any suggestion of reviewers is well taken with due gratitude and appreciation as a future scope. This is kept outside the purview of the present article, otherwise the focus of the present investigation might have shifted. Currently, the authors are engaged in exploring the effect of the present findings on complete path from a source node to destination nodes under various routing protocols. We are also trying to supplement the existing routing protocols with more information with the finding of the present work.

\section{References}

[1] M. Gerla, J. T. C. TSAI. Multicluster, mobile, multimedia radio network. IEEE Journal of Wireless Networks, 1(3):255-265, 1995.

[2] R. HEKMAT. Ad-hoc Networks:Fundamental Properties and Network Topologies. Springer, AA Dordrecht, The Netherlands, 2006.

[3] T. CAmp, J. Boleng, V. Davies. A survey of mobility models for ad hoc network research. Wireless Communications and Mobile Computing (WCMC): Special Issue on Mobile Ad Hoc Networking: Research, Trends and Applications, 2(5):483-502, 2002.

[4] Q. Zheng, X. Hong, S. RaY. Recent advances in mobility modeling for mobile ad hoc network research. In ACM Southeast Regional Conference, pages 70-75. ACM New York, NY, USA, 2004.

[5] E. Hyytï̈, P. LAssila, J. ViRTAMo. A markovian waypoint mobility model with application to hotspot modeling. In IEEE International Conference on Communications, volume 3, pages 979-986, 2006.

[6] C. Bettstetter. Topology properties of ad hoc networks with random waypoint mobility. Mobile Computing and Communication Review, 7(3):50 52, July 2003.

[7] P. Lassila, E. Hyytiä, H. Koskinen. Connectivity properties of random waypoint mobility model for ad hoc networks. In MedHoc-Net, ille de Porquerolles, pages 159-168, 2005.

[8] X. Hong, M. Gerla, G. Pei, C. C. Chiang. A Group Mobility Model for Ad Hoc Wireless Networks. Workshop on Modelling and Simulation of Wireless and Mobile Systems (MSWiM), pages 53-60, 1999.
[9] D. B. Johnson, D. A. Maltz. Dynamic source routing in ad hoc wireless networks. Mobile Computing, 353:153-181, 1996.

[10] C. E. Perkins, P. Bhagwat. Highly dynamic destination-sequenced distance-vector routing (DSDV) for mobile computers. Computer Communication Review, 24:234-244, 1994.

[11] C. E. PERKINS. Ad-hoc on-demand distance vector routing. In 2nd IEEE Workshop on Mobile Computing Systems and Applications, pages 90-100, 1999.

[12] F. BAi, N. Sadagopan, A. Helmy. IMPORTANT: A framework to systematically analyze the impact of mobility on performance of routing protocols for ad hoc networks. In IEEE INFOCOM, pages $825-835,2003$.

[13] F. BAI, G. Bhaskara, A. Helmy. BRICS: a building-block approach for analyzing routing protocols in ad hoc networks-a case study of reactive routing protocols. Computer Communication Review, 34(3):57-70, 2004.

[14] G. Bhaskara, A. Helmy, S. Gupta. Micromobility protocol design and evaluation: A parameterized building block approach. In Vehicular Technology Conference VTC-2003, volume 3, pages 2019-2024, 2003.

[15] J. Hamilton. Time Series Analysis. Princeton University Press, Princeton, New Jersy, 1994.

[16] P. J. Brockwell, R. A. DAVIS. Time Series: Theory and Methods. Springer-Verlag, New York, 1987.

[17] S. Basu, A. MukherJee, S. Klivansky. Time series models for internet traffic. In INFOCOM, volume 2, pages 611-620, March 1996.

[18] J. P. Singh, P. DuTTA. Temporal behavior analysis of mobile ad hoc network with different mobility patterns. In ICAC3 '09: International Conference on Advances in Computing, Communication and Control, pages 696-702, 2009. ACM.

[19] J. P. Singh, P. DuTTA. Temporal modeling of node mobility in mobile ad hoc network. Journal of Computing and Information Technology, 18(1):19-29, 2010.

[20] K. Xu, M. Gerla, S. BAE. Effectiveness of RTS/CTS handshake in IEEE 802.11 based ad hoc networks. Ad Hoc Networks, 1:107-123, 2003.

[21] University of Bonn. Bonnmotion - a mobility scenario generation and analysis tool. http://www.cs.uni-bonn.de/IV/bomonet/ BonnMotion.htm.

[22] J. Yoon, M. LiU, B. Noble. Random waypoint considered harmful. In INFOCOM 2003, pages 1312-1321, 2003.

[23] S. MCCAnNE, S. Floyd. ns Network Simulator. http://www.isi.edu/nsnam/ns/. 


\author{
Received: February, 2010 \\ Revised: July, 2011 \\ Accepted: September, 2011 \\ Contact addresses: \\ Jyoti Prakash Singh \\ Department of Information Technology \\ National Institute of Technology \\ Patna-800005, Bihar, India \\ e-mail: jyotip.singh@gmail.com \\ Paramartha Dutta \\ Department of Computer \\ and System Sciences \\ Visva-Bharati University \\ West Bengal, India \\ e-mail: paramartha.dutta@gmail.com
}

JyOTI PRAKASH SINGH did his B. Tech in Computer Science and Technology from Kalyani Government Engineering College, West Bengal, India in the year 2000. He completed his M.Tech in Information Technology in the year 2005 from Sikkim Manipal Institute of Technology, Sikkim, India. He is currently an Assistant Professor in the Department of Information Technology in National Institute of Technology, Patna, Bihar, India. He has been visiting/guest faculty in Kalyani Government Engineering College, West Bengal, India. He has co-authored five books. He has more than 26 research publications in various national and international journals and conference proceedings. His research interests include sensor ad hoc network, network security and secret sharing. He is Member of IEEE Computer Society USA, Computer Society of India, International Association of Engineers, Hong Kong and International Association of Computer and Information Technology, Singapore.

PARAMARTHA DUTTA did his Bachelors and Masters in Statistics from Indian Statistical Institute, Kolkata, India in 1988 and 1990 respectively. Subsequently, he did his Master of Technology in Computer Science in 1993 from Indian Statistical Institute, Kolkata, India. He completed his Ph. D. in Engineering in 2005 from Bengal Engineering and Science University, Shibpur, India while he was in service. He is currently a Professor in the Department of Computer and System Sciences, Visva-Bharati University, Santiniketan, West Bengal, India. He has been visiting/guest professor in (i) University of Kalyani, Nadia, West Bengal, India, (ii) Bengal Engineering and Science University, Shibpur, West Bengal, India, (iii) University of Tripura, India. He has co-authored 4 books and has 1 edited book to his credit. Apart from this he has more than 100 research publications in various national and international journals and conference proceedings and book chapters. His research interests include evolutionary computation, soft and intelligent computing, pattern recognition and mobile computing. Dr. Dutta is a Fellow of Optical Society of India and Institution of Electronics and Telecommunication Engineering. He is a Life Member of Indian Science Congress Association, Computer Society of India, Indian affiliate of International Association of Pattern Recognition, Indian Society for Technical Education, Advanced Computing and Communication Society and International Association of Engineers, Hong Kong. He is also a Member of Association for Computing Machinery, USA and IEEE Computers Society USA. 